\title{
Study of the short-circuit behaviour of wind farms
}

\author{
M.P. Comech, I. Franco, S. Martín, S. Borroy, M. García-Gracia \\ CIRCE-Departamento de Ingeniería Eléctrica de la Universidad de Zaragoza \\ C/ María de Luna nº 3, 50018 Zaragoza (España) \\ Email: mggracia@posta.unizar.es
}

\begin{abstract}
The aim of this paper is the assessment of the wind generation influence on the voltage quality. A simple network has been simulated by PSS/E [1].

The short circuit behaviour of the wind turbines has been analysed by means of simulation. To achieve it, several studies have been carried out. The purpose of these studies is to select the best options to modify the existing network as adding substations, branches, transformers, as well as calculate correctly the influence of the wind generation on power quality.
\end{abstract}

\section{Key words}

Wind energy, short circuit behaviour, wind farm connection.

\section{Introduction}

The influence of wind energy on the grid behaviour depends mainly on the wind power and the short circuit power at the point of connexion [2].

Nowadays, the electrical companies are specially worried about the behaviour of the wind generators against short circuits. In order to study this behaviour it is necessary to make a complete study by means of dynamic simulation. $\mathrm{PSS} / \mathrm{E}$ is a tool widely used by the electrical companies worldwide.

In this paper a complete study of the behaviour of a wind farm against different faults and the influence of the different parameters from the network in this behaviour is made.

The voltage drops directly affect the provision continuity. A voltage drop in a point of the network takes place, when the voltage of one or more phases falls suddenly below an established limit (generally 90\%) and recovers after a determined time, which oscillates between $10 \mathrm{~ms}$ and several seconds.
The wind energy is included in the regime of the activity of production of electrical energy in special regime, in opposition to the installation that conforms the ordinary regime. This special regime was systematized in Law 5554/1997. RD 436/2004 of 12th March incorporates the requirements of voltage drops. The system operator must develop the operation procedure that regulates the minimum requirements to fulfil by the special regime installations.

\section{Machine model on PSS/E}

A widely used squirrel cage induction generator model has been considered [3]. The induction machine is modelled using a Thevenin equivalent as it can be seen in Fig 1.

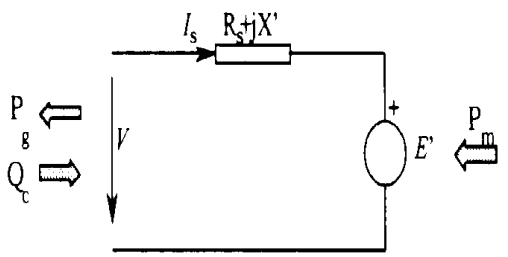

Fig 1. Thevenin equivalent

In this model, $R_{s}$ is the stator resistance and, $X$ ' the transient reactance. The voltage source $E$ ' is a complex variable which governs by means of the following equation differential:

$$
\frac{d E^{\prime}}{d t}=j 2 \pi f_{0} s \cdot\left(V_{r}^{\prime}-E^{\prime}\right)-\frac{1}{T_{0}{ }^{\prime}}\left[E^{\prime}-j\left(X-X^{\prime}\right) I_{s}\right]
$$

Where $f_{0}$ is the electrical frequency of the network (in Hertz), $s$ is the slip of the generator and $I_{s}$ the stator current (current consumed by the generator). Vr' is the rotor feeding voltage, whose value is zero in the case of a machine with the rotor short-circuited.

The constants of the previous equation are referred to the equivalent circuit of an asynchronous machine in 
permanent state shown in Fig 2. Next these constants are detailed.

- $\mathrm{X}^{\prime}$ is the transient reactance of the machine (short circuit reactance):

$$
X^{\prime}=X_{s}+\frac{X_{r} \cdot X_{m}}{X_{r}+X_{m}}
$$

- $\mathrm{T}_{0}$, is the transient constant of time at open circuit

$$
T_{0}{ }^{\prime}=\frac{X_{r}+X_{m}}{2 \cdot \pi \cdot f_{0} \cdot R_{r}}
$$

- $\mathrm{X}$ is the generator reactance at open circuit

$$
X=X_{s}+X_{m}
$$

Where $X_{m}$ is the magnetizing reactance of the generator and $\mathrm{Rr}$ the resistance of the rotor. $R_{S}, R_{r}, X_{s}, X_{r}$ and $X_{m}$ are obtained from the values of the equivalent circuit of an asynchronous machine shown in Fig 2.

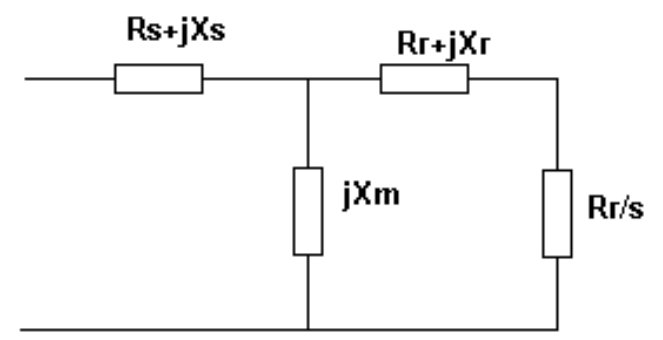

Fig 2. Single-phase equivalent of the asynchronous machine in permanent state.

\section{A. Harvest energy from the wind}

The wind turbine capacity for extract the energy from the wind depends on three factors:

-- The wind power available. Its value depends on the wind distribution, which is a characteristic of the wind farm location.

-- The power curve of the machine which is function of the type of machine, and

-- The good behaviour of the machine to respond to fluctuations in the wind speed. This is a characteristic of turbine technology.

The mechanical power extracted from the wind is:

$$
P=\frac{1}{2} \rho \cdot A \cdot C p(\lambda) \cdot V_{w}^{3}
$$

Where:

- $\quad \operatorname{Cp}(\lambda)$ is the power coefficient which depends on the specific speed $\lambda$. It is an adimensional magnitude.

- $\quad \rho$ is the air density in $\mathrm{kg} / \mathrm{m}^{3}$, commonly the used value is $1,225 \mathrm{~kg} / \mathrm{m}^{3}$.

- $\quad A$ is the area swept by the turbine blades in $\mathrm{m}^{2}$.

- $\mathrm{V}_{\mathrm{w}}$ is the wind speed in $\mathrm{m} / \mathrm{s}$.

\section{B. Power Curve of the Wind Turbine}

The power curve represents the electrical power produced by the wind turbine for each wind speed in permanent regime.

This curve is a data given by the manufacturer. The power curve is a basic characteristic of the machine.

The power curve allows calculating the energy produced by the machine from the wind distribution of the zone.

Several sections can be defined in the power curve:

- From calm $(0 \mathrm{~m} / \mathrm{s})$ to a speed from 3 to $5 \mathrm{~m} / \mathrm{s}$ the machine does not produce energy.

- From the starting speed ( 3 to $5 \mathrm{~m} / \mathrm{s}$ ) to the nominal speed ( $\sim 12$ to $14 \mathrm{~m} / \mathrm{s}$ ), there is a section of increasing power. Due to the blade stall in generators CSCF, the curve has a characteristic section called commonly "hump", in which is reached the maximum power, followed by a section of higher speeds and smaller power produced.

- When the wind speed is greater than a value around $25 \mathrm{~m} / \mathrm{s}$, the generators stop to produce electrical energy to avoid problems of mechanical overload.

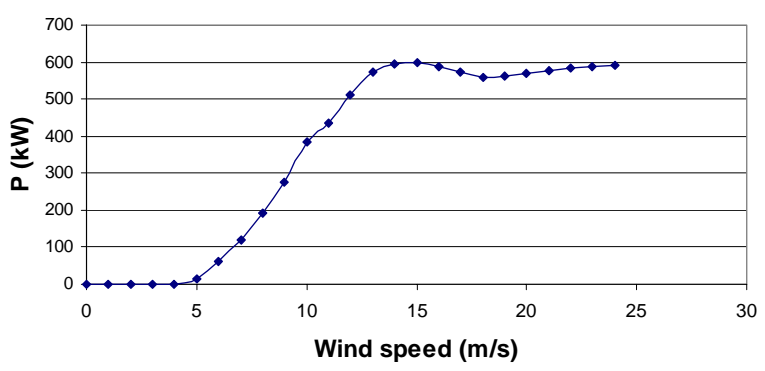

Fig 3. Power curve.

\section{Mechanical set}

The wind kinetic energy is caught by the blades of the wind turbine. The blades are made of composite material which allows to obtain a high resistance with a reduced weight. The blades are the most flexible element of the set, but for this kind of studies infinite rigidity is usually considered.

The blades turn at an almost constant speed of approximately 20-30 rpm. Due to the fact that this speed is clearly insufficient to produce electrical energy, a multiplier is used to elevate the speed of 40 to 100 times the speed of the blades, so that nominal speed is obtained in the output axis (around $1500 \mathrm{rpm}$ ).

The axes are jointed by means of a clutch Due to the important rigidity of the axes (blades and generator) and the multiplier, infinite rigidity is considered for the set. Only the clutch elasticity and damping can be nondespicable, mainly in the greatest machines. 


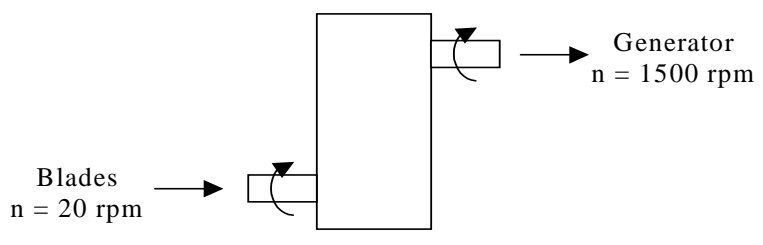

Fig 4. Gearbox layout.

The rest of the frictions, (viscous and static) are very small and its existence does not affect the dynamic behaviour of the generator for this study.

In this paper it has been considered a equivalent inertia moment equals to the sum of inertias of the generator, multiplier, bushing and blades, all referred the axis of the generator:

$$
J_{\text {eq }}=J_{\text {gen }}+J_{\text {gearbox }}+J_{\text {hub }}^{\prime}+J_{\text {blades }}^{\prime}
$$

Where

$$
J_{\text {blades hhub }}^{\prime}=J_{\text {blades }+ \text { hub }} \cdot\left(\frac{1}{r_{r}}\right)^{2}
$$

and

$$
r_{r}=\frac{n_{\text {generator }}}{n_{\text {blades }}}
$$

In stability studies, the fundamental variable is the inertia constant $H$, that gives an idea of the electromechanical response time of the machine before transitory. It is referred to the power base of the machine, and can be defined as:

$$
H=\frac{\frac{1}{2} \cdot J \cdot\left(\frac{2 \cdot \pi \cdot f_{\text {grid }}}{p}\right)^{2}}{S_{b}}
$$

where

- $\quad H$ is the inertia constant of the machines, in s (or MJ/MVA)

- $\quad J$ is the inertia moment of the machine referred to the alternator axis, in $\mathrm{Kg} \times \mathrm{m}^{2}$.

- $f_{\text {grid }}$ is the frequency bases of the network, in Hertz.

- $\quad p$ is the number of poles pairs of the machine.

- $S_{b}$ is the power base of the machine on VA.

As it can be appreciated, $H$ is the relation between the kinetic energy of the generator at the synchronism speed and the power base of the machine.

\section{Study description}

The influence of the grid parameters can be analyzed by means of a simple network. This single network is contrastable, and it permits us the control of the different parameters of the network in an easy way. Furthermore, the simulation time is significantly reduced.

The Fig 5 shows the network under test. The BUS 1 is the "boundary bus" which connects to the external network. The sum of the inertia of the external machines is very high, and then the external network has been modelled as a generator with infinite inertia.

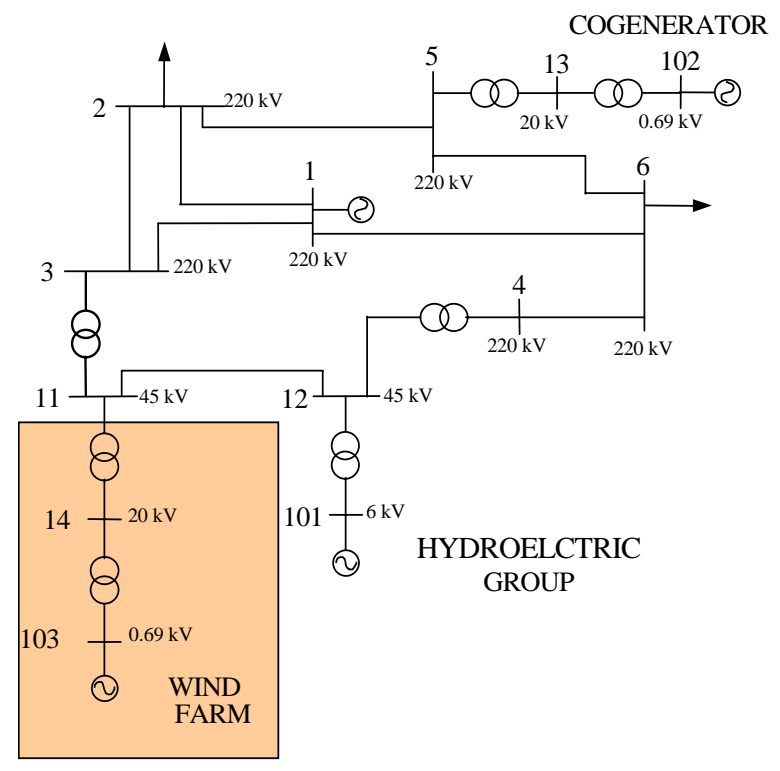

Fig 5. Equivalent network.

With the aim of consider the most unfavourable situation, the squirrel-cage induction turbines have been connected to the BUS 11. Moreover, a $15 \mathrm{MW}$ hydroelectric group, an $8 \mathrm{MW}$ co-generation system and two $15 \mathrm{MW}$ loads complete the network under test.

In this reduced network the behaviour before network faults has been studied. For the accomplishment of the network faults in PSS/E it is necessary to introduce a reactive component of shunt admittance to ground.

The made simulations present the following landmarks:

- Flat simulation until $\mathrm{t}=0,8 \mathrm{~s}$. PSS/E initiates its simulation starting from the stationary state from the values obtained in the load flow

- In $\mathrm{t}=0.8 \mathrm{~s}$ the fault is produced, and the voltage falls.

- In $t=1 \mathrm{~s}$ the fault is cleared and the simulation continues.

Three studies have been carried out:

- Influence of the wind power installed

- Influence of the voltage drop, and

- Influence of the short circuit power in the point of common coupling (PCC) 


\section{A. Influence of the wind power installed}

In this study all the parameters have remained constant with the exception of the installed power. Three studies have been realized; the number of wind turbines of the wind farm has been modified to obtain a total power installed of 6, 12 and 15 MW.

In order to make the simulation of the short circuits, in this study the same value of ground impedance has been introduced, and in each case a different voltage drop in the PCC has been obtained.

In Fig 6 it can be observed as the depth of the voltage drop raises when increasing the installed wind power.

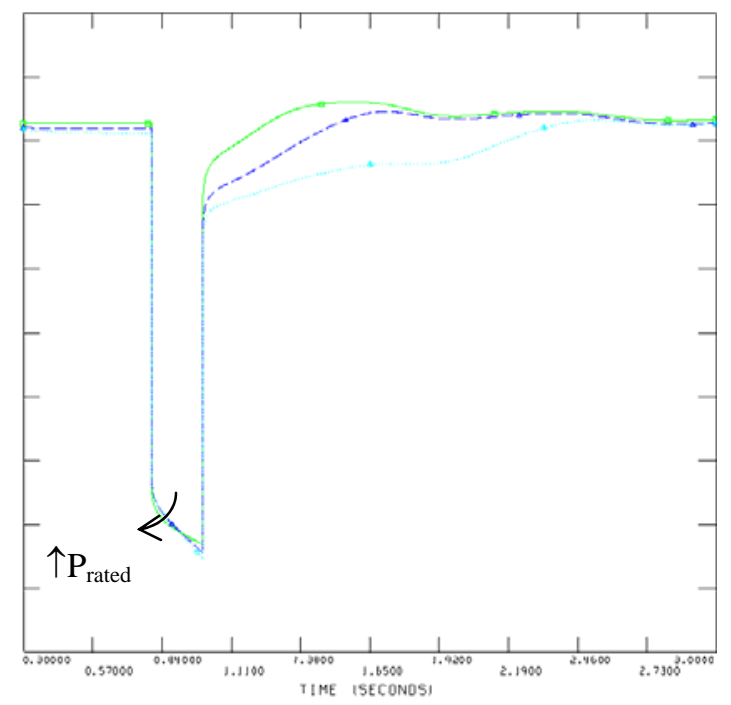

Fig 6. Installed power influence

TABLE I. Installed power influence

\begin{tabular}{|c|c|}
\hline & Voltage drop \\
\hline $\mathbf{6} \boldsymbol{M W}$ & $39.861 \%$ \\
\hline 12 $\boldsymbol{M W}$ & $40.667 \%$ \\
\hline $\mathbf{1 5} \boldsymbol{M W}$ & $41.203 \%$ \\
\hline
\end{tabular}

\section{B. Influence of the voltage drop}

In the analysis shown next, several short circuits have been simulated in the network with the $6 \mathrm{MW}$ wind farm. Fig 7 shows the depth of the different voltage drops, and Fig 8 the power evolution in each case.

As it could be expected, the diminution produced in the generated power when the voltage drop happens, increases when its depth is increased.

In addition, the time of recovery is greater in the case of the deepest voltage drops.

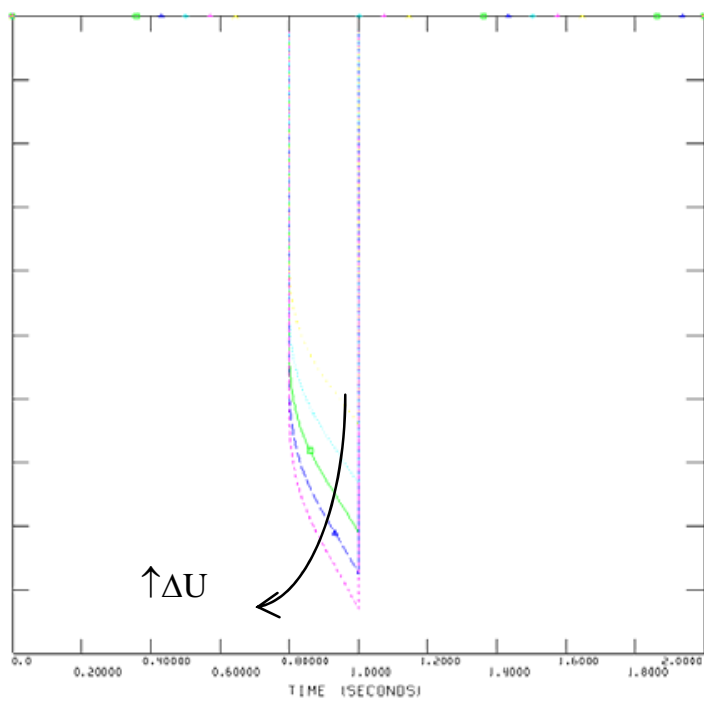

Fig 7. Voltage drop depth influence

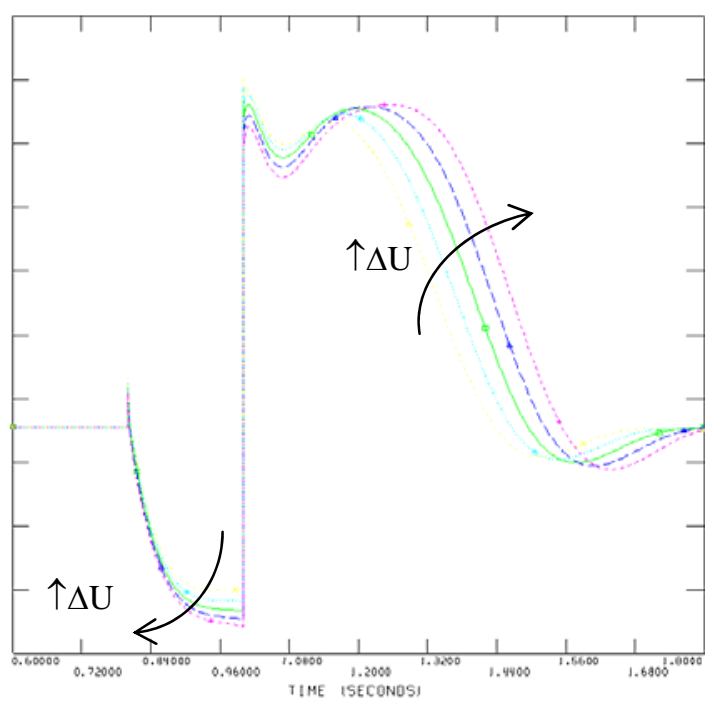

Fig 8. Voltage drop depth influence

C. Influence of the short circuit power in the point of common coupling (PCC)

One of the most important parameters in this type of studies is the short circuit power in the point of common coupling. In the previous norm (R.D. of 1985) the power installed in a network bus could not surpass the $20 \%$ of the power of short circuit in this point. This value still continues being a reference value, reason why the observation of the behaviour before the variation of this parameter is an important subject.

For these reasons, the study of the influence of short circuit power has been made. 


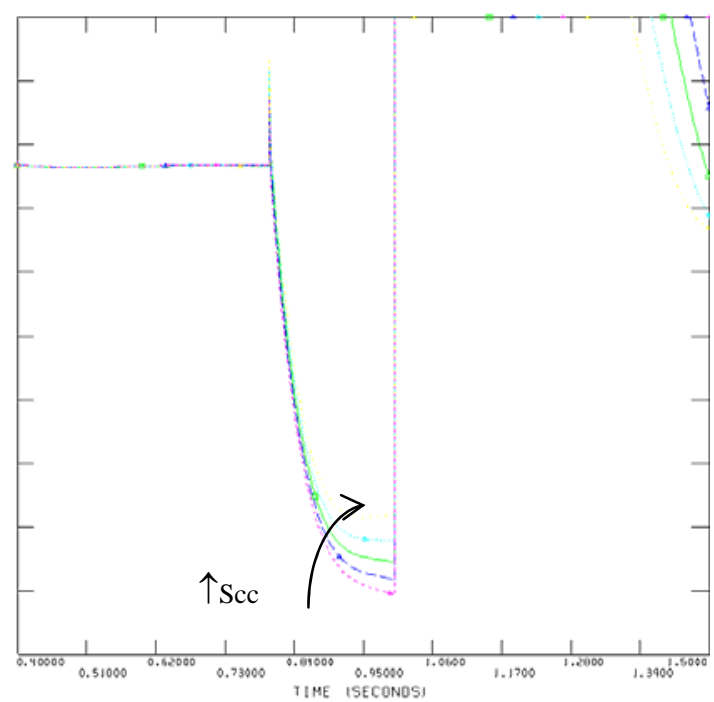

Fig 9. Short circuit power influence.

In Fig 9 the voltage evolution is shown. When the short circuit power in the PCC increases the depth of the drop diminishes, as it could be assumed.

Fig 10 shows the power evolution for the studied cases. When increasing the power of short circuit the diminution of the power in the voltage drop is smaller, and also the time of recovery.

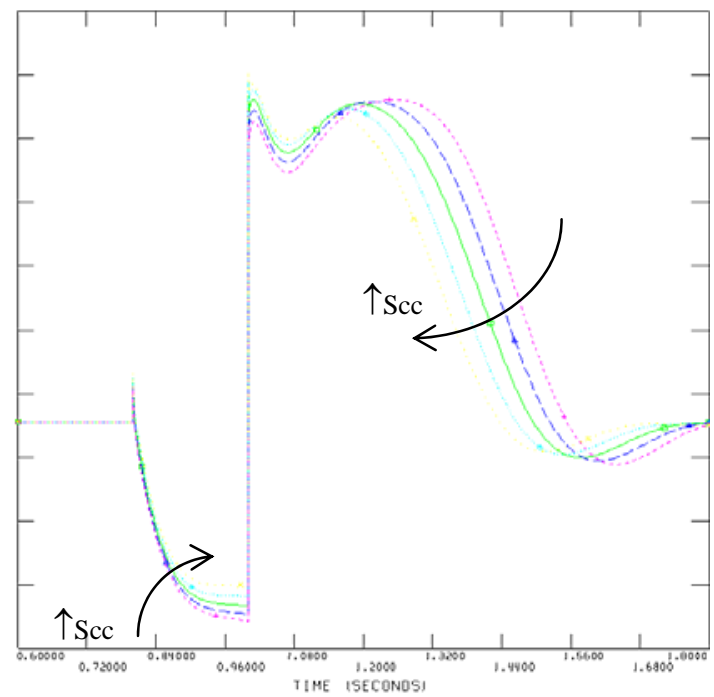

Fig 10. Short circuit power influence.
TABLE II. Short circuit power influence

\begin{tabular}{|c|c|}
\hline & Voltage drop \\
\hline 5 Ssc & $64.215 \%$ \\
\hline $\mathbf{1 . 6 7}$ Ssc & $66.99 \%$ \\
\hline Ssc & $69.29 \%$ \\
\hline $\mathbf{0 . 7 1}$ Ssc & $71.23 \%$ \\
\hline $\mathbf{0 . 5 5}$ Ssc & $72.86 \%$ \\
\hline
\end{tabular}

\section{Conclusions}

The short circuits and voltage drops study must be systematized due to the next norms modifications relative to special regime generators.

There are many parameters that influence in the behaviour of the electrical network with connected wind farms.

This paper analyzes the influence of three of them: The installed wind power, the depth of the voltage drop and the power of short circuit in the point of common coupling. The norm has special preoccupation for the behaviour of the networks before voltage drops, specially for the behaviour of the wind farms, therefore, the study of this behaviour would have to be systematized.

\section{References}

[1] PSS/E Power System Simulator for Engineering, Power Technologies Inc, version 26.2.3.

[2] D. García García, M.P. Comech, M. Sanz, J.B. Arroyo, M. García-Gracia. "Study of wind power limit for a network bus”. ICREPQ’03, Vigo (Spain), 2003.

[3] Yao-Nan Yu, "Electric Power System Dynamics", Academic Press, 1983. 